\title{
"Like For Like" Reconstruction of Heel Pad with Medial Plantar Artery Flap-Functional and Aesthetic Outcomes in A Series of 19 Cases
}

\author{
Narayanamurthy Sundaramurthy ${ }^{1}$ Surya Rao Venkata Mahipathi ${ }^{1}$, Alagar Raja Durairaj ${ }^{1}$ \\ Anand Prasath Jayachandiran ${ }^{1}$, Shalini Ranipet Selvaa ${ }^{2}$
}

${ }^{1}$ Department of Plastic and Reconstructive Surgery, Saveetha Medical College, Saveetha Nagar, Thandalam, Kanchipuram District, Tamil Nadu, India

2Saveetha Medical College, Saveetha Nagar, Thandalam,

Kanchipuram District, Tamil Nadu, India

\begin{abstract}
Address for correspondence Narayanamurthy Sundaramurthy, MS, MRCS, MCh, Department of Plastic and Reconstructive Surgery, Saveetha Medical College, Saveetha Nagar, Thandalam, Kanchipuram District, Tamil Nadu 602105, India (e-mail: nanathesurgeon@gmail.com).
\end{abstract}

Indian J Plast Surg 2021;54:358-361.

\begin{abstract}
Keywords

- heel reconstruction

- medial plantar artery flap

- versatile medial plantar axis

Heel pad loss can cause serious problems in weight-bearing and locomotion. The medial plantar artery (MPA) flap is a suitable "like for like" replacement. Nineteen patients whose heels were reconstructed with MPA flap between July 2015 and February 2020 were studied. All patients were assessed based on flap survival, functionality, and patient satisfaction. Loss of heel pad was due to diabetic ulcer (11), trauma (6), tumor (1), and unstable scar (1). The largest flap measured $9 \times 7 \mathrm{~cm}$. Sixteen flaps were done as fasciocutaneous flaps and three in combination with abductor hallucis muscle $(\mathrm{AbdH})$. All the flaps survived. The average functional scores at $6,12,18$, and 24 months were 86.86, 89.62, 89.38 and 97.33 based on AOSAS-AH score. Average patients' satisfaction was $8.7 / 10$. To conclude, the MPA system is a versatile vascular axis providing "like for like" tissue for heel pad reconstruction which is reliable and durable.
\end{abstract}

\section{Introduction}

The complex anatomy of heel pad allows it to bear weight, resist shearing forces, and act as shock absorber. Tissue loss at the heel is three-dimensional requiring dead space obliteration in addition to vascularized cover. Instep flap, first described by Harrison and Morgan in 1981, ${ }^{1}$ provides "like for like" vascularized tissue to reconstruct heel. ${ }^{2,3}$ The versatility of the medial plantar artery (MPA) axis allows various customizations. Abductor hallucis muscle $(\mathrm{AbdH})$ muscle can be used to fill the dead space, while the fasciocutaneous part covers it. In this article, we are presenting our functional results of MPA flap for heel reconstruction.

published online September 17, 2021
DOI https://doi.org/

$10.1055 / \mathrm{s}-0041-1735424$ ISSN 0970-0358

\section{Patients and Methods}

Nineteen patients who underwent heel pad reconstruction with MPA flap between July 2015 and February 2020 at our institute were retrospectively studied. Institute ethical committee clearance was obtained. Demographic features, dexterity, etiology, and size of the defect were recorded preoperatively. The presence of palpable dorsalis pedis artery (DPA) and posterior tibial artery (PTA) pulsations in a foot without collapse of arches were considered optimal to elevate a flap, based on MPA. Preoperatively, a handheld Doppler was used to mark the perforators from the superficial branch of MPA. Surgeries were done under regional anesthesia and

\section{(C) 2021. Association of Plastic Surgeons of India.}

This is an open access article published by Thieme under the terms of the Creative Commons Attribution-NonDerivative-NonCommercial-License, permitting copying and reproduction so long as the original work is given appropriate credit. Contents may not be used for commercial purposes, or adapted, remixed, transformed or built upon. (https://creativecommons.org/licenses/by-nc-nd/4.0/).

Thieme Medical and Scientific Publishers Pvt. Ltd. A-12, 2nd Floor, Sector 2, Noida-201301 UP, India 
tourniquet control. After debridement, proximally based sensate MPA flap was harvested to reconstruct heel. Plane of dissection remained deep to plantar fascia ( - Fig. 1). Secondary defect was covered with split-thickness skin graft (SSG). Postoperatively, compression garment with a silicone sheet and customized offloading footwear were provided. Physiotherapy and partial weight-bearing started from 4 to 5 weeks and total weight-bearing from 8 weeks.

During follow-up, return of protective sensations was tested using 10G Semmes-Weinstein monofilament. Functional assessment was done every 6 months following surgery. Two-point discrimination (2PD) was assessed in nondiabetic patients. The functionality of the foot was assessed based on American Orthopaedic Foot and Ankle SocietyAnkle and Hindfoot Score (AOFAS-AHS) ${ }^{4}$ every 6 months till 2 years, and patient satisfaction concerning aesthetics was graded on a visual analog scale of 0 to 10.3

\section{Results}

Etiologies of the 11 male and 8 female patients were diabetic foot disease (11), posttraumatic defects (6) ( - Fig. 2A), tumor (1) ( - Fig. 2B), and unstable scar (1). The average age was 52.74. The longest follow-up was for 51 months. The largest flap that was harvested was $9 \times 7 \mathrm{~cm}$ and the smallest $4 \times 3 \mathrm{~cm}$. In 16 patients, MPA flaps were harvested as fasciocutaneous flaps, and in 3 along with AbdH muscle. The average duration of surgery was 160 minutes for fasciocutaneous flaps and 210 minutes when AbdH muscle was included. Postoperatively, all flaps survived. Venous congestion was seen in three flaps. The first recovered by letting out of few in setting sutures. The second flap was salvaged with negative pressure wound therapy. The third flap had distal $3 \mathrm{~cm}$ loss, which was managed with dressings and secondary suturing. Eight nondiabetic patients regained protective sensation in the flap 4 weeks following surgery. Of the 15 patients who were followed-up for more than 6 months, ulcer recurrence was seen in one of the diabetic foot patient. Two patients had temporary loss of great toe sensation at 4 weeks, which normalized by 20 weeks. Average static $2 \mathrm{PD}$ was $9 \mathrm{~mm}$ at 3 months ( $3 \mathrm{~mm}$ more than the opposite side) and $6 \mathrm{~mm}$ at 6 months. AOFAS-AH scoring was done for 15 patients at 6 months, 13 at 12 months, 8 at 18 months, and 6 at 24 months. The average score at $6,12,18$, and 24 months were $86.87,89.62,89.38$, and 97.33 . The average aesthetic patient satisfaction outcome was 8.7/10.
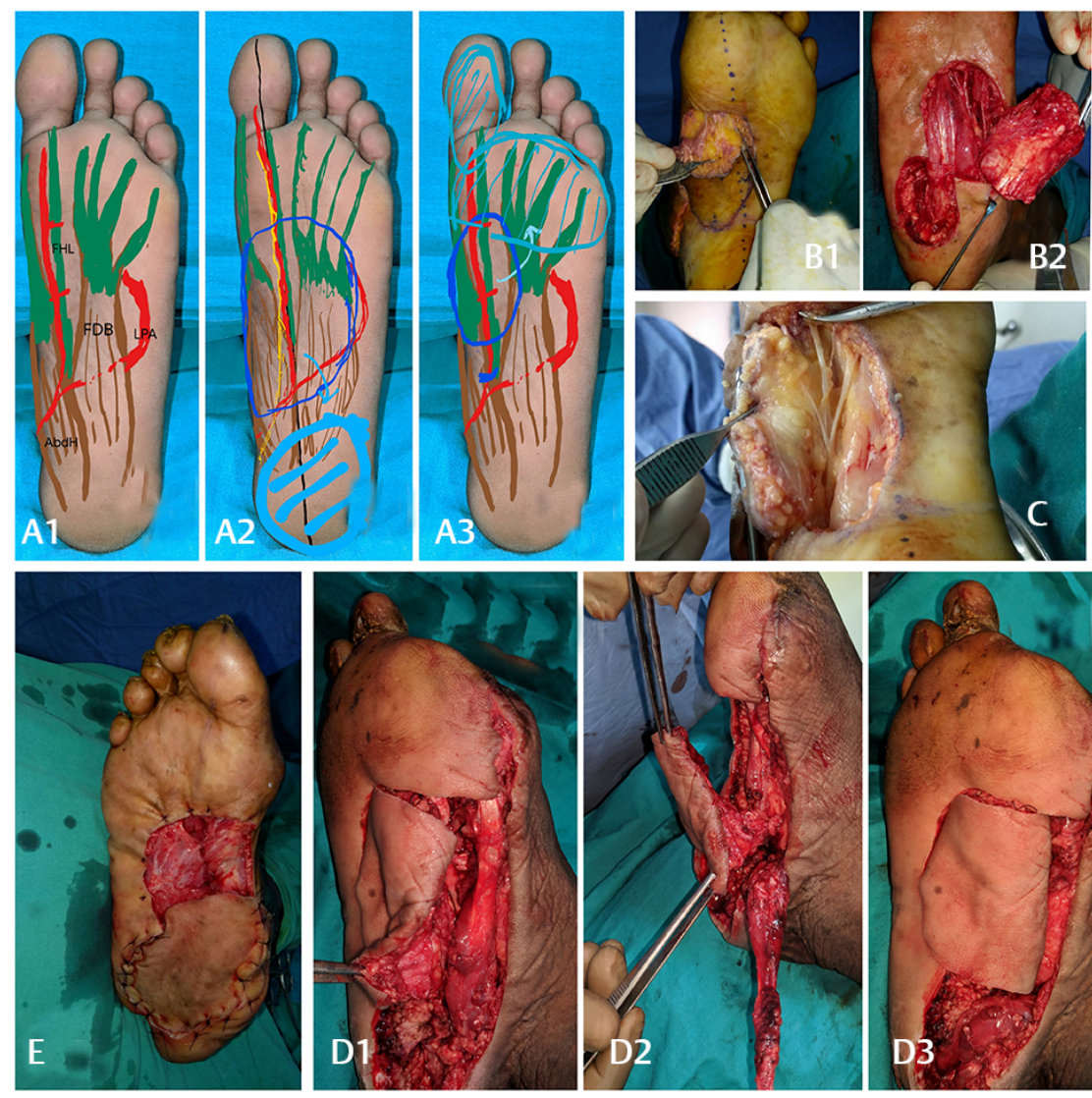

Fig. 1 (A) Marking of medial plantar artery (MPA) flap. A1, the relationship of vascular axis with abductor hallucis (AbdH), flexor hallucis longus (FHL), flexor digitorum brevis (FDB), and lateral plantar artery (LPA). A2, marking of the proximally based flap. Blackline-a line connecting calcaneum and head of great toe, light blue-a probable defect in the heel, dark blue-flap margins. A3, marking of distally based flap. (B) Flap elevation B1, distal incision and identification of distal superficial branch of MPA, shown with Adson's forceps; B2 flap elevated with vascular pedicle preserving nerves to toes. (C) Intraneural dissection of cutaneous branch of medial plantar nerve. (D) Use of AbdH muscle to fill dead space in the heel. D1, retraction of MPA fasciocutaneous flap with exposure of AbdH muscle. D2, elevation of the fasciocutaneous flap and AbdH muscle. D3, after muscle transposition and inset into the defect. (E) Preparation of bed for split-thickness skin graft (SSG) by approximating AbdH and FDB muscles. 

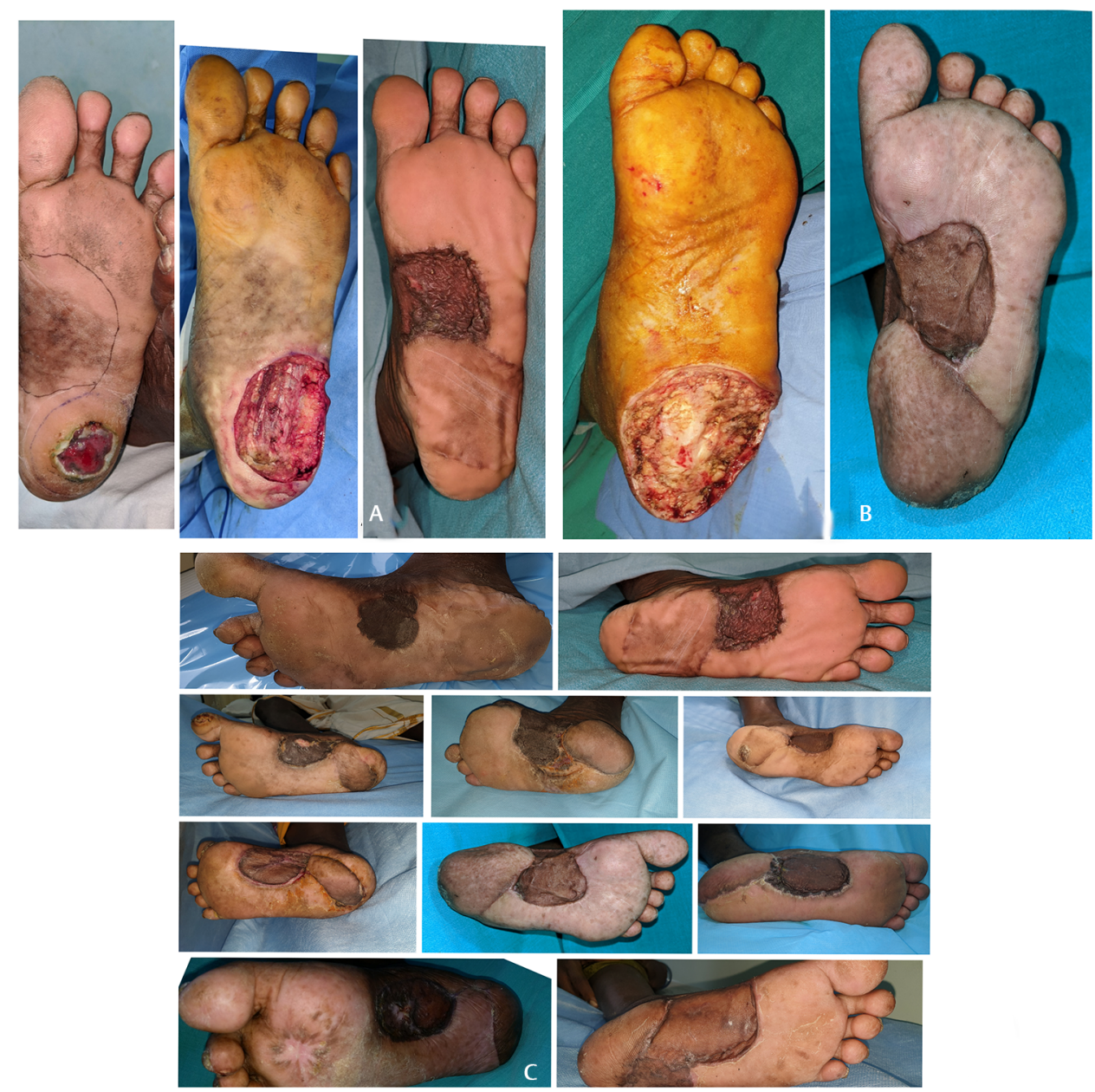

Fig. 2 (A) Reconstruction of heel following posttraumatic defect by medial plantar artery (MPA) fasciocutaneous flap. (B) Reconstruction of heel following melanoma excision A, ulcerative growth. B, after excision by the general surgery team. C, 12 months follow-up. (C) Long-term results of MPA flaps.

\section{Discussion}

MPA, the nondominant branch of PTA, divides into superficial and deep branches ${ }^{6-8}$ approximately 2.5 to $3.5 \mathrm{~cm}$ from its origin, deep to AbdH muscle. Based on superficial branch of MPA running in the medial intermuscular septum (MIS), instep flap can be elevated as either a proximally based flap or a distally based flap. ${ }^{9,10} \mathrm{AbdH}$ muscle, a type 2 muscle supplied proximally by branches from the deep branch of MPA and distally by the superficial branch of MPA, ${ }^{6}$ can be used to resurface smaller defects in the medial side of the heel as muscle transposition flap or in combination with the fasciocutaneous component to fill dead space ( - Fig. 1D). Thus, medial plantar vascular axis can be a source for various flaps and their modifications (-Fig. 3 ).

During flap harvest, exsanguination of the foot is not preferred. The patient is brought to the edge of the operating table with the surgeon at the leg end of the table.

Flap donor site morbidities are considered as one of the drawbacks of MPA flap. ${ }^{11-13}$ This can be reduced by approximating $\mathrm{AbdH}$ and flexor digitorum brevis (FDB) muscles to form a good bed for better graft uptake ( - Fig. 1E). We had to do a secondary SSG in two patients.

As 2PD values in diabetic patients can be variable due to neuropathy and compression at tarsal tunnel, ${ }^{14}$ we did not use this parameter in diabetics. The average 2PD among nondiabetic patients reached comparable levels with the opposite foot (same site) by 6 months ( $9 \mathrm{~mm})$, which is similar to those reported by Rinkel et al. ${ }^{15}$

The average AOFAS-AH score at 6 months was 86.86 and kept improving. The lowest score at 12 months in a patient was due to cellulitis in leg during assessment. At 18 months, one patient scored only 43 . He had osteomyelitis of third toe phalanges for which toe amputation was done elsewhere. He developed splaying of the foot due to probable loss of transverse metatarsal ligaments and developed an ulcer at the grafted donor site. After ray amputation, recreation of the arch and total contact cast application, his ulcer healed in 8 weeks. At 24 months, the average score was 97.33 , which meant our patients achieved near-normal stance and gait. Aesthetically, based on the visual analog scale of 0 to 10 , our patients gave an average score of 8.7 ( - Fig. 2 C). 


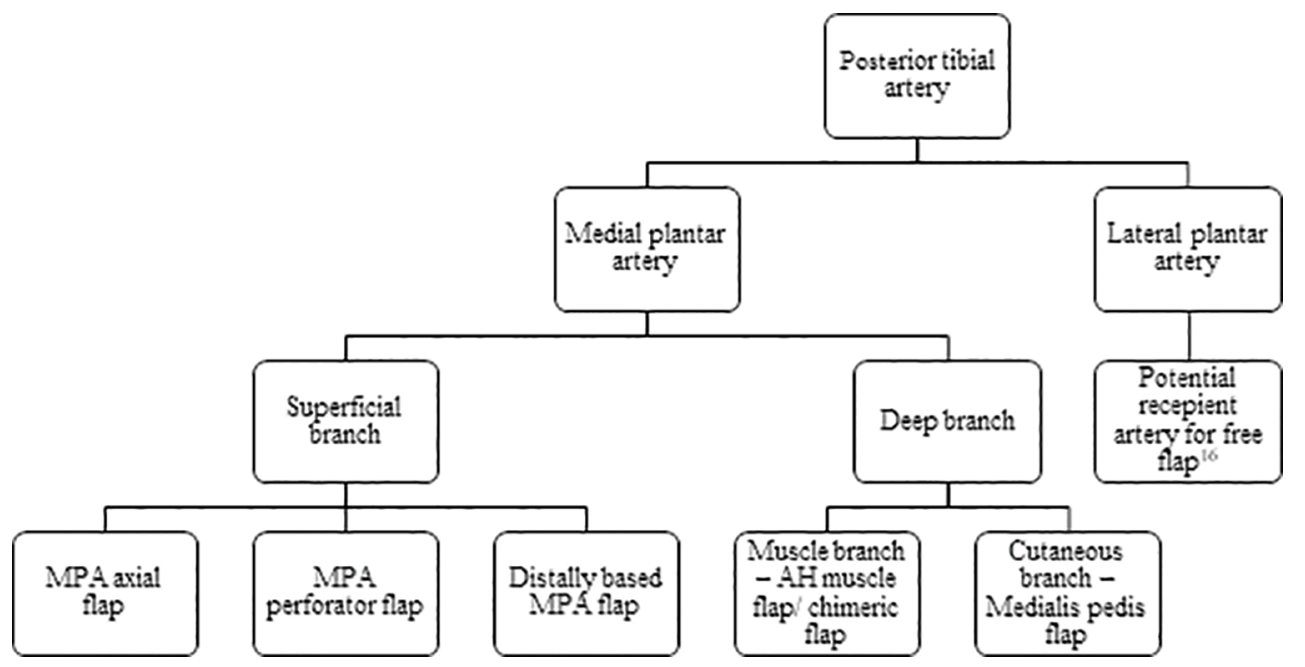

Fig. 3 Medial plantar vascular axis and possible flaps.

\section{Conclusion}

MPA flap is a reliable and durable "like for like" tissue replacement for heel reconstruction whenever the instep region is nonweight-bearing in a nonischemic foot. The versatile MPA axis enables customization of the flap, according to three-dimensional needs of the defect.

\section{Funding/Grants}

Nil.

\section{Conflict of Interest}

None declared.

\section{References}

1 Harrison DH, Morgan BD. The instep island flap to resurface plantar defects. Br J Plast Surg 1981;34(3):315-318

2 Schwarz RJ, Negrini JF. Medial plantar artery island flap for heel reconstruction. Ann Plast Surg 2006;57(6):658-661

3 Gu JX, Huan AS, Zhang NC, et al. Reconstruction of heel soft tissue defects using medial plantar artery island pedicle flap: clinical experience and outcomes analysis. J Foot Ankle Surg 2017;56(2):226-229

4 Khai Luen K, Wan Sulaiman WA. Functional outcomes after heel pad reconstruction: a review of 7 cases. J Foot Ankle Surg 2017;56(5):1114-1120

5 Dolan RT, Butler JS, Murphy SM, Cronin KJ. Health-related quality of life, surgical and aesthetic outcomes following microvascular free flap reconstructions: an 8-year institutional review. Ann R Coll Surg Engl 2012;94(1):43-51
6 Rodriguez-Vegas M. Medialis pedis flap in the reconstruction of palmar skin defects of the digits: clarifying the anatomy of the medial plantar artery. Ann Plast Surg 2014;72(5):542-552

7 Low OW, Sebastin SJ, Cheah AEJ. A review of pedicled perforator flaps for reconstruction of the soft tissue defects of the leg and foot. Indian J Plast Surg 2019;52(1):26-36

8 Macchi V, Tiengo C, Porzionato A, et al. Correlation between the course of the medial plantar artery and the morphology of the abductor hallucis muscle. Clin Anat 2005;18(8):580-588

9 Scaglioni MF, Rittirsch D, Giovanoli P. Reconstruction of the heel, middle foot sole, and plantar forefoot with the medial plantar artery perforator flap: clinical experience with 28 cases. Plast Reconstr Surg 2018;141(1):200-208

10 Guillier D, Campisi C, Krähenbühl SM, Raffoul W, di Summa PG. Bipedicled distally based medial plantar artery perforator flap for forefoot reconstruction: a case report. Microsurgery 2020;40(4):497-500

11 Siddiqi MA, Hafeez K, Cheema TA, Rashid HU. The medial plantar artery flap: a series of cases over 14 years. J Foot Ankle Surg 2012;51(6):790-794

12 Yang D, Yang JF, Morris SF, Tang M, Nie C. Medial plantar artery perforator flap for soft-tissue reconstruction of the heel. Ann Plast Surg 2011;67(3):294-298

13 Wan DC, Gabbay J, Levi B, Boyd JB, Granzow JW. Quality of innervation in sensate medial plantar flaps for heel reconstruction. Plast Reconstr Surg 2011;127(2):723-730

14 Periyasamy R, Manivannan M, Narayanamurthy VBR. Changes in two point discrimination and the law of mobility in diabetes mellitus patients. J Brachial Plex Peripher Nerve Inj 2008;3:3

15 Rinkel WD, Aziz MH, Van Deelen MJ, et al. Normative data for cutaneous threshold and spatial discrimination in the feet. Muscle Nerve 2017;56(3):399-407

16 Chen Y-C, Scaglioni MF. The lateral plantar artery as recipient vessel for microsurgical lateral plantar forefoot reconstruction. Case Report International Microsurgery Journal 2017;1(2):2 